\title{
REFLEXIONES SOBRE ÉTICA DE INVESTIGACIÓN EN SERES HUMANOS
}

\begin{abstract}
Alberto Perales ${ }^{1, a}$
\section{RESUMEN}

Con el objetivo de plantear la necesaria reflexión moral sobre la responsabilidad personal que asume cada investigador cuando realiza investigación científica en seres humanos, se presenta un ensayo de temática científica y moral, utilizando información de la literatura científica y ética pertinentes. En torno a los conceptos de ética heterónoma y ética autónoma, se plantea que, en última instancia y allende el consentimiento Informado y los cursos de adiestramiento sobre ética en investigación, la conducta del/de la investigador/a dependerá de su propia responsabilidad moral puesta a prueba en diversos contextos situacionales. Se utilizan dos modelos explicativos para comprender esta dinámica, el de normalidad en salud mental y el del desarrollo moral del hombre. Se concluye que el proceso de investigación en seres humanos constituye una actividad científica y moral que, dependiendo de diversas situaciones de conflicto de interés, pondrá siempre a prueba los controles morales del investigador.
\end{abstract}

Palabras clave: Ética en investigación; Mala conducta científica; Moral; Consentimiento informado (fuente: DeCS BIREME).

\section{REFLECTIONS ABOUT RESEARCH ETHICS IN HUMANS}

\begin{abstract}
In order to propose the necessary moral reflection about the personal responsibility that each researcher assumes when he performs scientific research in human beings, an essay of moral and scientific themes is presented, using information from the relevant scientific and ethical literature. Around the concepts of heteronomous and autonomous ethics, it is proposed that ultimately and beyond informed consent and training courses on research ethics, the behavior of the researcher will depend on his/her own moral responsibility, tested in different situational contexts. Two explanatory models are used in order to understand this dynamic, the one of normality in mental health and the one of moral development of men. We conclude that the research process in human beings is a scientific and moral activity that, depending in various situations of conflicts of interest, will always test the researcher's moral controls.
\end{abstract}

Key words: Ethics, research; Scientific misconduct; moral; Informed consent (source: MeSH NLM).

\section{INTRODUCCIÓN}

Sócrates estaba convencido que el ser humano era bueno y que aquél que mal actuaba solo lo hacía por ignorancia. Desde que Ezekiel Emanuel publicara ¿Qué hace que la investigación clínica sea ética? (1), los investigadores hemos tenido criterios prácticos para disminuir la probabilidad, siempre presente y en contra de nuestras mejores intenciones, de afectar a las personas que participan como sujetos de experimentación en nuestras investigaciones.

Sumado a ello, las pautas internacionales específicas (2-4), el caudal de publicaciones sobre el tema, y, en nuestro país, la asignación de la responsabilidad del control de los Ensayos Clínicos al Instituto Nacional de Salud (INS) ${ }^{(5)}$, así como la aparición del Código de Ética y Deontología del Colegio Médico del Perú -articulado relativo al Trabajo de Investigación, Sección Segunda, Título I, Capítulo 6 - (6) han aportado sustento teórico suficiente para que todo investigador conduzca investigación científica en seres humanos dentro de los adecuados marcos éticos.

Al estar disponible tal conocimiento para todo profesional que desee realizar investigación científica en salud, debemos preguntarnos: ¿Por qué, entonces, aún se aprecian casos de violación de los principios éticos en investigación en seres humanos?

Cabe aquí recordar la conocida reflexión de Heráclito: Por lo tanto, es un deber seguir lo que es común a todos.

\footnotetext{
1 Instituto de Ética en Salud, Facultad de Medicina, Universidad Nacional Mayor de San Marcos. Lima, Perú

a Médico Psiquiatra, Doctor en Medicina.
} 
Pero, a pesar de que el logos* es común, viven los más como si poseyeran un entendimiento particular.

Una de las posibles respuestas a esta interrogante podría ser: Porque todos los investigadores reconocen las reglas éticas generales pero algunos las interpretan de modo diferente, en virtud de sus valores y experiencias personales. Y otra, Porque los investigadores conocen las reglas éticas a nivel intelectual pero no las internalizan a nivel afectivo. Un investigador puede saber mucho de ética pero no necesariamente vivenciarla como responsabilidad personal, recordemos que la ética no se aprende adecuadamente informándose sino vivenciándola. Por eso, Apel, -como señalaba Maliandi en la Maestría Internacional de Bioética, Lima 20022003, UNMSM -vio la necesidad de Complementar las exigencias propias del principio de universalidad (en nuestro caso las normas señaladas para la investigación en seres humanos) con las exigencias que derivan de las responsabilidades particulares (en nuestro caso la de los investigadores)" (7).

\section{EL ESTUDIO DE LA CONDUCTA HUMANA}

Conviene señalar que, desde el punto de vista del estudio del comportamiento humano, la conducta de cada investigador no dependerá exclusivamente de lo que sabe sino también, de muchos otros factores complejos. Para clarificar, aunque sea de modo muy general esta dinámica, nos apoyaremos en dos modelos explicativos, el de normalidad en salud mental y el del desarrollo moral del ser humano. Por problemas de espacio, lo haremos en forma muy resumida.

\section{EL MODELO DE NORMALIDAD EN SALUD MENTAL}

Perales, León y Mezzich ${ }^{(8)}$, propusieron un modelo para explicar la conducta humana normal y patológica. La tesis plantea que todo ser humano tiene áreas normales y anormales en su personalidad o, si se prefiere otras terminologías, áreas no conflictivas y conflictivas, o maduras e inmaduras. Así consideradas, la normalidad o anormalidad absolutas resultan utopías clínicas. La normalidad o anormalidad reales, dependen, más bien, del balance resultante, positivo o negativo, de la interacción entre ambas partes. El modelo, aplicado a la práctica, permite comprender por qué algunos seres humanos, considerados normales bajo diferentes criterios de evaluación, pueden, en circunstancias particulares, desarrollar conductas puntualmente anormales o desadaptadas, por ejemplo, cuando su área de inmadurez es específicamente estimulada (por ambiente corrupto de trabajo, ocasión de ganancias ilícitas especialmente si la persona está bajo presiones económicas personales, y muchas otras).

\section{EL DESARROLLO DE LA PERSONALIDAD Y MORAL DEL SER HUMANO.}

Ortiz ha planteado que el proceso de formación de la personalidad del homo sapiens, incluyendo su desarrollo moral, se inicia desde el nacimiento, en la medida que la información social que su ambiente le provee es codificada en su neocortex. El niño irá gradualmente absorbiendo los valores de su cultura y modulándolos de acuerdo con sus propias experiencias vitales ${ }^{(9)}$. En tal proceso, la moralización del ser humano permanece vigente durante toda la vida, pudiendo el hombre, tener la posibilidad, de acuerdo con su libertad personal, de modificar su conducta hasta el último de sus días.

Resumiendo, y sobre la base de estos dos modelos, podríamos decir que la conducta moral del investigador dependerá, en gran medida, de su propio desarrollo moral y de las circunstancias en que se dé su acción investigativa.

\section{¿POR QUÉ SE REQUIERE QUE LA INVESTIGACIÓN SEA ÉTICA?}

Resnik ${ }^{(10)}$ ha señalado varias razones para ello:

- Las normas éticas promueven los objetivos de investigación, por medio de la búsqueda de la verdad y del conocimiento así como por el control del error. Por ejemplo, prohíbe la falsificación de la información y el uso de datos falsos;

- Promociona los estándares éticos y valores esenciales para el trabajo colaborativo y la solidaridad en los equipos de investigación, generando la mutua confianza entre sus miembros y protegiendo la autoría de los estudios;

- Las normas convierten a los investigadores en responsables legales ante el público, especialmente cuando los proyectos son financiados por el Estado. La opinión pública necesita confiar en la integridad de los investigadores para continuar dando su apoyo;

- Muchas de las normas de investigación promocionan otros valores, tales como el de responsabilidad social, los derechos humanos, el bienestar de los animales, el valor de la salud, y otros.

En definitiva, todas las normas, nacionales e internacionales en ética de investigación, se orientan directamente a proteger a los seres humanos que nos permiten utilizar sus organismos como unidades

*A propuesta del autor del presente artículo. 
de experimentación; sin embargo, al protegerlos, indirectamente también protegen al investigador y a la propia institución donde se realiza el estudio.

Sorprendentemente, cuando esta dinámica de protección y autoprotección parecía clara, Trochim señala con acierto, que nuestra sociedad atraviesa actualmente por un periodo de profundos cambios en la comprensión de lo ético, de tal nivel, que obliga a una revisión profunda del impacto de sus normas. Estas, que teóricamente vigilan y evitan que los pacientes sean utilizados como "conejillos de Indias", complejizan su aplicación cuando, por ejemplo, en el campo oncológico, grupos de pacientes desesperados por la condición terminal de su enfermedad se asocian en grupos legalmente constituidos, para ofrecerse a la ciencia exactamente como eso, como "conejillos de indias", en una última y desesperada esperanza de detener el avance de su mal ${ }^{(11)}$.

Por otro lado, si a pesar de los obstáculos, el campo de la ética de la investigación viene consolidándose, hay dos áreas sobre las que conviene insistir: que los seres humanos no son los únicos sujetos de experimentación a los que hay que proteger; $y$, que las condiciones sociales de desigualdad facilitan que hayan seres humanos en mayor riesgo de ser explotados.

\section{ÉTICA DE LA INVESTIGACIÓN EN ANIMALES}

La ética de la investigación, para ser coherente con sus bases morales, ha debido extenderse a todo ser sintiente, habiéndose creado los Comités de Ética de Investigación en Animales o Committee on Animal Research and Ethics (CARE). De ellos, uno de los más connotados es aquel que pertenece a la Asociación Psicológica Americana (12). El objetivo fundamental de su función reside en delimitar claramente el propósito científico del estudio que debe estar plenamente justificado para utilizar animales de experimentación, especialmente en lo relativo al balance daño/beneficio. Exigencia de aprobación es que sus potenciales resultados sean de clara utilidad para el bien de la humanidad o de la especie estudiada. Solo bajo tales condiciones debiera avalarse el riesgo, daño o sufrimiento al que se someterá a los animales seleccionados. La conclusión es que en investigación con animales debe también salvaguardarse un nivel responsable, recomendándose el uso de guías específicas para conducir los estudios éticamente.

\section{ÉTICA DE INVESTIGACIÓN EN POBLACIONES VULNERABLES}

El Consejo Internacional de Organizaciones de Ciencias Médicas (CIOMS de sus siglas en inglés), define a las personas vulnerables, como: (Guideline 13) Aquellas que son relativa o absolutamente incapaces de proteger sus propios intereses. Individuos incluidos en esta categoría presentan recortada su libertad para dar su consentimiento o declinar de hacerlo.... (Guidelines 14 y15) incluyendo niños y personas que, a causa de trastornos mentales o de conducta muestran incapacidad de dar su consentimiento. Cuando esta situación ocurre, se requiere la participación de su representante legal. Otros grupos vulnerables lo constituyen los jóvenes que son subordinados de un grupo jerárquico de mayores (estudiantes de medicina o enfermería, personal de laboratorios farmacéuticos, miembros de las Fuerzas Armadas, pacientes psiquiátricos, poblaciones indígenas, ancianos institucionalizados, etc). En estos casos, especiales consideraciones éticas deben ser tomadas $^{(3)}$.

En países como el Perú, además, debe considerarse que poblaciones vulnerables por razón de origen nativo, extrema pobreza o bajo o inexistente nivel educativo, reaccionan ante la autoridad del médico o del personal de salud, con actitudes de extrema pasividad y sumisión, ampliando así el margen de posibles manipulaciones en su contra. Una vez más, en estos casos, la moral responsable del propio investigador es fundamental, pues la ética de la investigación no se ejercita con la exclusiva aplicación del Consentimiento Informado condición necesaria pero no suficiente - sino durante todo el proceso del estudio que solo termina cuando este es publicado.

\section{OTROS FACTORES}

Hans Selye, el lúcido creador de la Teoría General de Adaptación y del concepto de estrés que tanto ha servido y sirve en medicina y salud, señala en uno de sus libros, que en su amplia experiencia de Director del Instituto de Medicina y Cirugía Experimental de la Universidad de Montreal, Canadá, hubo de entrevistar a múltiples postulantes que deseaban trabajar con él como investigadores. De tales experiencias concluye que, en general, Los científicos suelen ser muy ambiciosos y competitivos.... Asimismo, postula que un investigador debe poseer algunas características personales esenciales: 1) Honestidad consigo mismo, lograda por introspección y auto-examen; 2) Autodisciplina mental, control de su mente orientándola a la acción efectiva; $y$, 3) Autodisciplina física, con imposición de un modo saludable de vida ${ }^{(13)}$.

Ya en lo práctico, podemos señalar que los problemas éticos suelen surgir cuando el investigador ingresa a una situación de conflicto de interés, en la cual, un 
interés secundario, usualmente vinculado con ganancia de poder, prestigio o dinero, vence a su interés primario por la ciencia y quiebra sus controles morales. Conviene recordar por ello que, desde Aristóteles, sabemos que toda ética es situacional.

Por ello, resulta realistamente deseable, como acertadamente señala Arboleda (14): Que a los investigadores se les debe capacitar también en ética de la investigación y que (estos) deben estar muy conscientes de las vulnerabilidades de sus posibles sujetos. Declaramos nuestro total acuerdo con tal propuesta, pero quisiéramos señalar que tal capacitación no debe ser de naturaleza exclusivamente cognoscitiva sino vivencial.

\section{ÉTICA AUTÓNOMA Y ÉTICA HETERÓNOMA}

Los términos autónomo y heterónomo derivan de vocablos griegos que significan independiente, el primero, y, distinto, de otro, el segundo. Inmanuel Kant (1724-1804) los planteó como conceptos básicos en el campo de la ética. Un hombre obra bien cuando basa su conducta en un principio universal - imperativo categórico - válido para todos los hombres, en todas las circunstancias, tiempo o lugar. El hombre ejerce, así, su voluntad racional, autónomamente. Pero, en la práctica, tal accionar estará éticamente limitado por la ética heterónoma, exigencia que procede de los otros - incluyendo las ideologías políticas y credos religiosos. Cuando la norma obedece no al deber en sí mismo, sino a fines u objetivos impuestos por los demás, la conducta resulta condicionada, no libre imperativo hipotético. De este modo, si actuamos en función de principios normativos ajenos a nuestra libre voluntad estaremos condicionados, obedeceremos a causas externas y, por tanto, de acuerdo con Kant, será imposible que nuestras acciones sean genuinamente buenas y correctas. Nuestro obrar debe ser libre, ajeno a condiciones, a imposiciones exteriores; debe ser fin en sí mismo. Por ello, para este autor, el obrar humano debe ser bueno y correcto, racionalmente justificado e internamente asumido. Y este buen obrar es lo que Kant considera como imperativo categórico, guiado por dos postulados:

- Obra de modo que puedas querer la máxima de tu acción como ley universal.

- Obra de modo que trates a la humanidad, tanto en tu persona como en la de cualquier otro, siempre como un fin y nunca como un medio.

A este respecto, debemos reconocer el valor de los argumentos Kantianos, aunque limitados por la realidad humana. El hombre actúa siempre mediado por posibles consecuencias, en un ambiente social que, querámoslo o no, lo condiciona. A ello debemos agregar lo postulado por Spinoza: que la naturaleza humana no solo es racional, pues también está impregnada de sentimientos, pasiones, deseos, miedos,... que en ocasiones se sumergen en lo irracional, absurdo o, incluso, aberrante. Por todo ello, el idealismo de Kant deberá encontrar su punto de equilibrio con la realidad social, en la que, para bien o para mal, nos desenvolvemos.

Planteado el problema en esta perspectiva, podemos resumir diciendo que la ética heterónoma es aquella que el individuo acepta en virtud de la influencia externa, generalmente vinculada con el sistema moral culturalmente impuesto en su sociedad o medio. Es decir, el sujeto acepta las normas morales que le enseñan sus otros significativos que teóricamente debieran regir las buenas conductas en su comunidad. De este modo, la moral heterónoma, urge la obediencia absoluta a los principios y normas, aun sin que estos sean bien comprendidos, bajo la tesis de que tal obediencia conducirá a las relaciones de buena convivencia y al desarrollo, no solo de la sociedad sino del propio individuo.

La ética autónoma, por el contrario, surge como resultado del proceso de reflexión personal sobre lo bueno y lo malo en la propia conducta. No se trata de hacer algo porque nos enseñaron que es bueno sino de hacerlo porque sentimos y estamos convencidos que lo es. La ética autónoma induce a las personas a la auto-reflexión y las conduce a evaluar y asumir la responsabilidad de sus actos. En la esfera moral, la autonomía implica una voluntad libre que, mediante la razón, muestra que ciertos principios morales son correctos.

Podría decirse que actuar exclusivamente por moral heterónoma, sería cumplir con los principios morales solo por temor al castigo o interés por el premio, mientras que hacerlo autónomamente es aceptar que el cumplimiento de los principios es un deber, y que, en última instancia, el hombre (el/la investigador/a) en situación de soledad y total responsabilidad es quien debe decidir sus actos.

\section{ASPECTOS FINALES}

Desde esta perspectiva y aplicada a los seres humanos que se desempeñan como investigadores científicos, las normas, los códigos, las leyes y otras exigencias de la comunidad científica, constituyen presiones deontológicas vinculadas a la ética heterónoma. Los diversos cursos de adiestramiento en ética de investigación implican ese perfil, y suelen ser muy útiles a 
condición de que no se descuide el desarrollo de la ética autónoma que, en última instancia, será la única que garantice la bondad moral de los actos del investigador durante todo el proceso que dure la investigación, pues, el hecho que el proyecto apruebe la evaluación de un Comité de Ética en Investigación, tan solo demuestra que el investigador o investigadores, tienen las mejores intenciones de actuar científica y moralmente en forma adecuada. Pero, de la intención a la obra en sí, el camino puede ser muy corto y recto o muy largo y tortuoso.

Finalmente, y en la misma perspectiva aristotélica de buscar soluciones intermedias - puntos de equilibrio - a los problemas morales, tampoco conviene que la ética heterónoma reglamente todo; reglamentar la libertad individual, incluso en materia de fe o pensamiento, resulta funesto, porque, como nuevamente recordamos a Spinoza, Legislarlo todo no limita las pasiones ni limita los sentimientos, incrementa los vicios.

\section{Conflictos de Interés}

El autor declara no tener conflictos de interés en la publicación de este artículo.

\section{REFERENCIAS BIBLIOGRÁFICAS}

1. Ezekiel E. ¿Qué hace que la investigación clínica sea ética? En: Pellegrini Filho A, Macklin R (eds). Investigación en sujetos humanos: experiencia internacional. Santiago de Chile: Programa Regional de Bioética, Organización Panamericana de la Salud; 1999.

2. World Medical Association (WMA). Declaration of Helsinki. ethical principles for medical research involving human subjects. 59th WMA General Assembly. Seoul, October 2008.

3. Council for International Organizations of Medical Sciences (CIOMS). International ethical guidelines for biomedical research involving human subjects. Geneva: World Health Organization; 2002.

4. World Health Organization (WHO). Handbook for good clinical research practice (GCP). Guidance for implementation. Geneva; WHO; 2002.

5. Instituto Nacional de Salud (INS). Reglamento de ensayos clínicos. Lima: INS; 2010.

6. Colegio Médico del Perú (CMP). Código de ética y deontología. Lima: CMP; 2007.

7. Apel L O. Teoría de la verdad y ética del discurso. Barcelona: Paidos; 1991.

8. Perales A, León R, Mezzich JE. Un modelo de normalidad en salud mental y clasificación multiaxial. An Salud Mental. 1989;5(1y2):11-28.

9. Ortiz P. Introducción a una psicobiología del hombre. $2^{\text {da }}$ ed. Lima: Universidad Nacional Mayor de San Marcos; 2010.

10. Resnik DB. What is ethics in research \& why is it important? Research Triangle Park, North Carolina: National Institute of Environmental Health Sciences/National Institute of Health; 2010.

11. Trochim W. The research methods knowledge base. $2^{\text {nd }}$ ed. Cincinnati, OH: Atomic Dog Publishing; 2000.

12. American Psychological Association (APA). Guidelines for ethical conduct in the care and use of animals. Washington DC: APA; 1996.

13. Selye H. From dream to discovery. On being a scientist. Toronto: McGraw-Hill Book Company; 1964. p.

14. Arboleda J. La investigación en sujetos humanos: poblaciones vulnerables. En: Pellegrini Filho A, Macklin $\mathrm{R}$ (eds). Investigación en sujetos humanos: experiencia Internacional. Santiago de Chile: Programa Regional de Bioética, Organización Panamericana de la Salud; 1999.

Correspondencia: Alberto Perales Cabrera

Dirección: Av. Grau 755. Lima 1, Perú.

Teléfono: (511) 6197000

Email: perales.alberto@gmail.com.pe

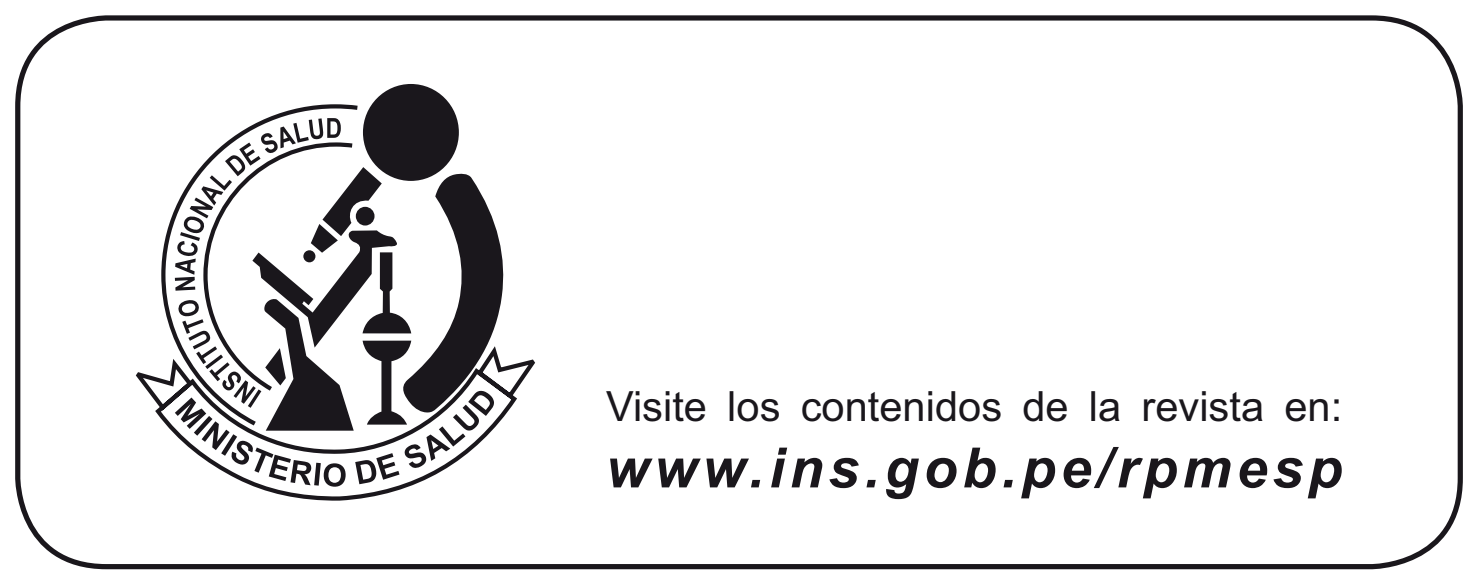

